\title{
Monotonicity of Löwner Operators and Its Applications to Symmetric Cone Complementarity Problems
}

\author{
Lingchen Kong*, Levent Tunçel ${ }^{\dagger}$, and Naihua Xiu ${ }^{\ddagger}$
}

(April 21, 2009; revised: July 22, 2010)

\begin{abstract}
We prove necessary and sufficient conditions for locally Lipschitz Löwner operators to be monotone, strictly monotone and strongly monotone. Utilizing our characterization of the strict monotonicity of Löwner operators, we generalize Mangasarian class of Nonlinear Complementarity Problem (NCP)-functions to the setting of symmetric cone complementarity problem. This affirmatively answers a question of Tseng [Math. Program. 83, 159-185(1998)].
\end{abstract}

Key words: Löwner operator, Euclidean Jordan algebra, Monotonicity, Symmetric cone complementarity problem, C-function

MSC2000 subject classification: Primary: 65K05, 90C33; secondary: 26B05

1. Introduction. In 1934, Löwner [15] defined a matrix-valued operator $G$, which is later called Löwner Operator by Sun and Sun [18], as

$$
G(x):=\sum_{i=1}^{n} g\left(\lambda_{i}(x)\right) u_{i} u_{i}^{T} \quad \text { if } \quad x=\sum_{i=1}^{n} \lambda_{i}(x) u_{i} u_{i}^{T}
$$

where $g$ is a real-valued scalar function, $\lambda_{i}(x)$ and $u_{i}(i=1,2, \cdots, n)$ are the eigenvalues and the corresponding eigenvectors of the symmetric matrix $x$, respectively. In the same paper, Löwner studied the operator-monotonicity of the operator $G$ in the setting of symmetric matrices where the partial order is induced by the cone of positive semidefinite matrices. These concepts and corresponding theoretical results have become fundamental ingredients in Matrix Analysis.

In 1984, Korányi [7] gave a full generalization of Löwner's theorem to the setting where the underlying algebra is a simple Euclidean Jordan algebra and the partial order is induced by the underlying symmetric cone. In the setting of Euclidean Jordan algebras, Löwner operator has recently attracted much attention. For example, Sun and Sun [18] established its differentiability and semismoothness; Baes [1] studied its convexity using techniques similar to Lewis $[12,13]$.

\footnotetext{
* Lingchen Kong, Department of Applied Mathematics, Beijing Jiaotong University, Beijing 100044, P. R. China; Department of Combinatorics and Optimization, Faculty of Mathematics, University of Waterloo, Waterloo, Ontario N2L 3G1, Canada (e-mail: konglchen@126.com)

${ }^{\dagger}$ Levent Tunçel, Department of Combinatorics and Optimization, Faculty of Mathematics, University of Waterloo, Waterloo, Ontario N2L 3G1, Canada (e-mail: ltuncel@math.uwaterloo.ca)

$\ddagger$ Naihua Xiu, Department of Applied Mathematics, Beijing Jiaotong University, Beijing 100044, P. R. China (e-mail: nhxiu@center.njtu.edu.cn)
} 
Note that operator-monotonicity of $G$ is different from the usual concept of monotonicity of $G$. The latter is much weaker, which plays a crucial role in the theory and algorithms for complementarity problems, variational inequality problems and equilibrium problems $[4,17]$. However, to the best of our knowledge, monotonicity of Löwner operator $G$ has not been considered even in the cases of the cone of positive semi-definite matrices and the second-order cone. An interesting and important question arises:

What is the connection between the monotonicity of the real-valued function $g$ and its corresponding Löwner operator $G$ ?

On another front, in the area of nonlinear complementarity problem (NCP), there is an elegant result of Mangasarian [16] which provides a very general tool for reformulation of NCPs based on Mangasarian class of NCP-functions which is defined as $\phi_{M}: \mathbb{R} \times \mathbb{R} \rightarrow \mathbb{R}$,

$$
\phi_{M}(a, b):=\theta(|a-b|)-\theta(a)-\theta(b),
$$

where $\theta: \mathbb{R} \rightarrow \mathbb{R}$ is strictly increasing with $\theta(0)=0$. The above function has attracted much attention, see, e.g., [4, 20]. In particular, Tseng [20] asked whether Mangasarian class of NCP-functions above can be generalized to complementarity functions (C-functions) for the semidefinite complementarity problems (SDCPs).

In this note, we prove that the locally Lipschitz Löwner operator $G$ is (strictly/strongly) monotone on the a Euclidean Jordan algebra $\mathcal{J}$ if and only if the scalar function $g$ is (strictly/strongly) increasing on $\mathbb{R}$. We also give an important application of the result which ties in the theorems of Löwner, Korányi and Mangasarian. Namely, we generalize Mangasarian class of NCP-functions to the case of symmetric cone complementarity problem (SCCP for short). Therefore, not only does our result answer Tseng's question in the affirmative, but it also establishes the positive answer in the more general and unifying setting of symmetric cones. Here, SCCP is to find a vector $x \in \mathcal{J}$ such that

$$
x \in K, F(x) \in K,\langle x, F(x)\rangle=0,
$$

where $F: \mathcal{J} \rightarrow \mathcal{J}$ is a continuous mapping. SCCP provides a simple, natural, and unified framework for various existing complementarity problems, such as the NCP, SDCP and the second-order cone complementarity problem (SOCCP); see, e.g., [3, 5, 6, 8, 11, 14, 21].

This note is organized as follows. In the next section, we establish the preliminaries. In Section 3, we prove our main equivalence result. As an application, we extend the Mangasarian class of NCP-functions in Section 4. We only briefly present our main results in this note, see the long version [9] for further details.

2. Preliminaries. A Euclidean Jordan algebra is a triple $(\mathcal{J},\langle\cdot, \cdot\rangle, \circ)(\mathcal{A}$ for short $)$, where $(\mathcal{J},\langle\cdot, \cdot\rangle)$ is a finite-dimensional inner product space over real field $\mathbb{R}$ and $(x, y) \mapsto$ $x \circ y: \mathcal{J} \times \mathcal{J} \rightarrow \mathcal{J}$ is a bilinear mapping which satisfies the following conditions:

(i) $x \circ y=y \circ x$ for all $x, y \in \mathcal{J}$,

(ii) $x \circ\left(x^{2} \circ y\right)=x^{2} \circ(x \circ y)$ for all $x, y \in \mathcal{J}$ where $x^{2}:=x \circ x$ and

(iii) $\langle x \circ y, z\rangle=\langle x, y \circ z\rangle$ for all $x, y, z \in \mathcal{J}$.

We call $x \circ y$ the Jordan product of $x$ and $y$. In addition, we assume that there is an element $e$ (called the identity element) such that $x \circ e=e \circ x=x$ for all $x \in \mathcal{J}$. Define the set of squares as $K:=\left\{x^{2}: x \in \mathcal{J}\right\}$. It is well known that $K$ is a symmetric cone. 
An element $c \in \mathcal{J}$ is idempotent if $c^{2}=c \neq 0$. It is also primitive if it cannot be written as a sum of two idempotents. A complete system of orthogonal idempotents is a finite set $\left\{c_{1}, c_{2}, \cdots, c_{k}\right\}$ of idempotents with $c_{i} \circ c_{j}=0(i \neq j)$ and $\sum_{i=1}^{k} c_{i}=e$. A complete system of orthogonal primitive idempotents is called a Jordan frame of $\mathcal{A}$. In the Euclidean Jordan algebra $\mathcal{A}$, for any element $x \in \mathcal{J}$, the degree of $x$ is the smallest positive integer such that the set $\left\{e, x, x^{2}, \cdots, x^{m}\right\}$ is linearly dependent, denoted by $\operatorname{deg}(x)$. The rank of $\mathcal{A}$ is defined as $\operatorname{rank}(\mathcal{A}):=\max \{\operatorname{deg}(x): x \in \mathcal{J}\}$. Thus, we have the following important spectral decomposition theorems.

Spectral Decomposition Type I (Theorem III.1.1, [2])) Let $\mathcal{A}$ be a Euclidean Jordan algebra. Then for $x \in \mathcal{J}$ there exist unique real numbers $\mu_{1}(x), \mu_{2}(x), \cdots, \mu_{\bar{r}}(x)$, all distinct, and a unique complete system of orthogonal idempotents $\left\{b_{1}, b_{2}, \cdots, b_{\bar{r}}\right\}$ such that $x=$ $\mu_{1}(x) b_{1}+\cdots+\mu_{\bar{r}}(x) b_{\bar{r}}$.

Spectral Decomposition Type II (Theorem III.1.2, [2])) Let $\mathcal{A}$ be a Euclidean Jordan algebra of rank $r$. Then for $x \in \mathcal{J}$ there exist a Jordan frame $\left\{c_{1}, c_{2}, \cdots, c_{r}\right\}$ and real numbers $\lambda_{1}(x), \lambda_{2}(x), \cdots, \lambda_{r}(x)$, the eigenvalues of $x$, such that

$$
x=\lambda_{1}(x) c_{1}+\lambda_{2}(x) c_{2}+\cdots+\lambda_{r}(x) c_{r} .
$$

We call (1) the spectral decomposition of $x$.

Note that the Jordan frame $\left\{c_{1}, c_{2}, \cdots, c_{r}\right\}$ and the unique complete system of orthogonal idempotents $\left\{b_{1}, b_{2}, \cdots, b_{\bar{r}}\right\}$ in the above theorems depend on $x$. Let $\sigma(x)$ be the set consisting of all distinct eigenvalues of $x$. It follows that $\sigma(x)$ contains at least one element and at most $r$. For each $\mu_{i}(x) \in \sigma(x)$, denoting $N_{i}(x):=\left\{j: \lambda_{j}(x)=\mu_{i}(x)\right\}$, we can verify that $\bar{r}$ is the number of elements in $\left\{b_{i}: \mu_{i}(x) \in \sigma(x)\right\}$ and $b_{i}=\sum_{j \in N_{i}(x)} c_{j}$.

Given a real interval $(a, b)$ with $a<b(a, b \in \mathbb{R} \cup\{-\infty\} \cup\{+\infty\})$, we denote by $\mathcal{J}(a, b)$ the set of all $x$ in $\mathcal{J}$ such that $x-a e, b e-x \in \operatorname{int}(K)$, and call it the open box in $\mathcal{A}$. Letting $g$ : $(a, b) \rightarrow \mathbb{R}$ be a real-valued function, for $x \in \mathcal{J}(a, b)$ with $x=\sum_{j=1}^{r} \lambda_{j}(x) c_{j}=\sum_{i=1}^{\bar{r}} \mu_{i}(x) b_{i}$, we define the corresponding Löwner operator as

$$
G(x):=\sum_{j=1}^{r} g\left(\lambda_{j}(x)\right) c_{j}=\sum_{i=1}^{\bar{r}} g\left(\mu_{i}(x)\right) b_{i} .
$$

When $g(t)$ is taken as $t_{+}:=\max \{0, t\}$ or $|t|(t \in \mathbb{R})$, respectively, we have

$$
x_{+}:=\sum_{i=1}^{r}\left(\lambda_{i}(x)\right)_{+} c_{i}, \quad|x|:=\sum_{i=1}^{r}\left|\lambda_{i}(x)\right| c_{i} .
$$

We recall the Peirce decomposition of Euclidean Jordan algebras. Let $\left\{c_{1}, c_{2}, \cdots, c_{r}\right\}$ be a Jordan frame of $\mathcal{A}$. For $i, j \in\{1,2, \cdots, r\}$, define the subspaces

$$
J_{i i}:=\left\{y \in \mathcal{J}: y \circ c_{i}=y\right\}, \text { and } J_{i j}:=\left\{y \in \mathcal{J}: y \circ c_{i}=\frac{1}{2} y=y \circ c_{j}\right\}, i \neq j .
$$

Theorem 1 (Theorem IV.2.1, [2]) Let $\mathcal{A}$ be a Euclidean Jordan algebra of rank $r$ and $\left\{c_{1}, c_{2}, \cdots, c_{r}\right\}$ be a given Jordan frame. Then $\mathcal{J}$ is the orthogonal direct sum of spaces $J_{i j}(i \leq j)$. Furthermore, 
(i) $J_{i j} \circ J_{i j} \subseteq J_{i i}+J_{j j}$;

(ii) $J_{i j} \circ J_{j k} \subseteq J_{i k}$, if $i \neq k$;

(iii) $J_{i j} \circ J_{k l}=\{0\}$, if $\{i, j\} \cap\{k, l\}=\varnothing$.

For each $x \in \mathcal{J}$, we define the corresponding Lyapunov transformation $L(x): \mathcal{J} \rightarrow \mathcal{J}$ by $L(x) y=x \circ y$ for all $y \in \mathcal{J}$. Thus, $L(x)$ is a symmetric operator with respect to the inner product in the sense that $\langle L(x) y, z\rangle=\langle y, L(x) z\rangle, \forall y, z \in \mathcal{J}$. We say two elements $x, y \in \mathcal{J}$ operator commute if $L(x) L(y)=L(y) L(x)$. For each $x \in \mathcal{J}$, define the quadratic representation of $x$ as $Q(x):=2 L^{2}(x)-L\left(x^{2}\right)$. Lemma X.2.2 [2] shows that the elements $x, y \in \mathcal{J}$ operator commute if and only if they share a common Jordan frame.

We end this section with the following theorem which is important in the subsequent analysis. Observing carefully the proof of Theorem 2.14 in [8], we found that it is still valid when "semismoothness" property of $G(\cdot)$ is weakened to "local Lipschitzian" property of $G(\cdot)$.

Theorem 2 If $g(\cdot)$ is locally Lipschitz on $(a, b)$, then the function $G(\cdot)$ is locally Lipschitz on $\mathcal{J}(a, b)$. Furthermore, the Clarke generalized Jacobian $\partial G(x)$ of $G(x)$ for every $x \in \mathcal{J}(a, b)$ is a set of symmetric linear operators from $\mathcal{J}$ into itself, and satisfies

$$
\bar{\partial} G(x) \supseteq \partial G(x) \supseteq \underline{\partial} G(x)
$$

with the sets $\bar{\partial} G(x)$ and $\underline{\partial} G(x)$ being given respectively by

$$
\bar{\partial} G(x):=\operatorname{conv}\left[\bigcup_{\left\{c_{1}, \cdots, c_{r}\right\} \in \mathcal{C}(x)} \partial_{c_{1}, \cdots, c_{r}} G(x)\right]
$$

and

$$
\underline{\partial} G(x):=\left\{2 \sum_{i \neq j, i, j=1}^{\bar{r}}\left[\mu_{i}(x), \mu_{j}(x)\right]_{g} L\left(b_{i}(x)\right) L\left(b_{j}(x)\right)+\sum_{i=1}^{\bar{r}} \partial g\left(\mu_{i}(x)\right) Q\left(b_{i}(x)\right)\right\},
$$

where $\partial_{c_{1}, \cdots, c_{r}} G(x):=\left\{2 \sum_{i \neq j, i, j=1}^{r}\left\{\left[\lambda_{i}(x), \lambda_{j}(x)\right]_{g}\right\} L\left(c_{i}\right) L\left(c_{j}\right)+\sum_{i=1}^{r} \partial g\left(\lambda_{i}(x)\right) Q\left(c_{i}\right)\right\}, \mathcal{C}(x)$ is the set consisting of all Jordan frames in the spectral decomposition type II of $x$, and

$$
\left[\tau_{i}, \tau_{j}\right]_{g}:\left\{\begin{array}{cc}
=\frac{g\left(\tau_{i}\right)-g\left(\tau_{j}\right)}{\tau_{i}-\tau_{j}} & \text { if } \tau_{i} \neq \tau_{j} \\
\in \partial g\left(\tau_{i}\right) & \text { if } \tau_{i}=\tau_{j} .
\end{array}\right.
$$

3. Monotonicity. This section establishes that the monotonicity of $g$ is equivalent to that of the Löwner operator $G$.

Theorem 3 Let $g$ be a locally Lipschitz function from $(a, b)$ into $\mathbb{R}$, and let $G$ be the corresponding Löwner operator from $\mathcal{J}(a, b)$ into $\mathcal{J}$. Then the following hold:

(a) $G$ is monotone on $\mathcal{J}(a, b)$ if and only if $g$ is monotone on $(a, b)$.

(b) $G$ is strictly monotone on $\mathcal{J}(a, b)$ if and only if $g$ is strictly monotone on $(a, b)$. 
(c) $G$ is strongly monotone with modulus $\mu>0$ on $\mathcal{J}(a, b)$ if and only if $g$ is strongly monotone with modulus $\mu>0$ on $(a, b)$.

Proof. Part (a): " $\Rightarrow$ " It is trivial from the definition of monotonicity.

" $\Leftarrow$ " Since $g(\cdot)$ is locally Lipschitz, by Theorem 2 the Clarke generalized Jacobian $\partial G(\cdot)$ exists. Since $g$ is monotone, the subdifferential $\partial g(t) \subseteq \mathbb{R}_{+}$for every $t \in(a, b)$. Hence, the conclusion follows immediately from Theorem 2 and Theorem 2.17 in [8].

Part (b): " $\Rightarrow$ " It is similar to the proof of Part (a).

" $\Leftarrow$ " For any $x, y \in \mathcal{J}(a, b)$, we consider the following two cases.

Case 1: If $x, y$ operator commute, then there is a Jordan frame $\left\{e_{1}, \cdots, e_{r}\right\}$ such that $x=$ $\sum_{i=1}^{r} x_{i} e_{i}, y=\sum_{i=1}^{r} y_{i} e_{i}$. Then, $x \neq y$ if and only if there exists an index $i \in\{1,2, \cdots, r\}$ such that $x_{i} \neq y_{i}$. By strict monotonicity of $g,\left\langle y_{i}-x_{i}, g\left(y_{i}\right)-g\left(x_{i}\right)\right\rangle>0$ for $y_{i} \neq x_{i}$. Thus, we derive that if $x \neq y$

$$
\begin{aligned}
\langle y-x, G(y)-G(x)\rangle & =\left\langle\sum_{i=1}^{r} y_{i} e_{i}-\sum_{i=1}^{r} x_{i} e_{i}, G\left(\sum_{i=1}^{r} y_{i} e_{i}\right)-G\left(\sum_{i=1}^{r} x_{i} e_{i}\right)\right\rangle \\
& =\sum_{i=1}^{r}\left\langle y_{i}-x_{i}, g\left(y_{i}\right)-g\left(x_{i}\right)\right\rangle\left\|e_{i}\right\|^{2}>0 .
\end{aligned}
$$

Case 2: If $x, y$ do not operator commute, then setting $h:=y-x$, we have $y=x+h$. For any $z$ in the convex hull $[x, y]$ of $x$ and $y$, it is easy to show that $z$ and $h$ do not operator commute either.

We will show $\langle h, V h\rangle>0$ for all $V \in \partial G(z)$ with $z \in[x, y]$ and $h=y-x$ when $x, y$ do not operator commute. By Theorem 2 and the definition of $\bar{\partial} G$, it suffices to demonstrate that $\langle h, V h\rangle>0$ for every $\left\{c_{1}, \cdots, c_{r}\right\} \in \mathcal{C}(z)$ and $V \in \partial_{c_{1}, \cdots, c_{r}} G(z)$. In this case, we take $z=$ $\sum_{i=1}^{r} \lambda_{i}(z) c_{i}$, and $V=2 \sum_{i \neq j, i, j=1}^{r} \nu_{i j} L\left(c_{j}\right) L\left(c_{i}\right)+\sum_{i=1}^{r} \nu_{i i} Q\left(c_{i}\right)$ with $\nu_{i j} \in\left\{\left[\lambda_{i}(z), \lambda_{j}(z)\right]_{g}\right\}$. For every $i, j \in\{1,2, \cdots, r\}$, we define $w_{i j}:=\min \left\{\left[\lambda_{i}(z), \lambda_{j}(z)\right]_{g}\right\}$. Then $\nu_{i j}-w_{i j} \geq 0$ and

$$
w_{i j}=\left\{\begin{array}{cc}
\frac{g\left(\lambda_{i}(z)\right)-g\left(\lambda_{j}(z)\right)}{\lambda_{i}(z)-\lambda_{j}(z)}>0 & \text { if } \quad \lambda_{i}(z) \neq \lambda_{j}(z), \\
w_{i i}=w_{j j} \geq 0 & \text { if } \quad \lambda_{i}(z)=\lambda_{j}(z),
\end{array}\right.
$$

by the strict monotonicity of $g$. Based on this, we further define

$$
W:=2 \sum_{i \neq j, i, j=1}^{r} w_{i j} L\left(c_{i}\right) L\left(c_{j}\right)+\sum_{i=1}^{r} w_{i i} Q\left(c_{i}\right) .
$$

As in the proof of Theorem 2.11 in [8], we conclude that $\langle h, V h\rangle-\langle h, W h\rangle \geq 0$. Thus, it suffices to verify $\langle h, W h\rangle>0$.

Consider Spectral Decomposition Type I of $z$, i.e., $z=\sum_{i=1}^{\bar{r}} \mu_{i}(z) b_{i}$ as in Section 2 with $N_{i}(z):=\left\{j: \lambda_{j}(z)=\mu_{i}(z)\right\}$ and $b_{i}:=\sum_{j \in N_{i}(z)} c_{j}$. It holds by Theorem 1 and the argument 
after Spectral Decomposition Type II that

$$
\begin{aligned}
h & =\sum_{i=1}^{r} h_{i} c_{i}+\sum_{1 \leq j<l \leq r} h_{j l} \\
& =\sum_{i=1}^{\bar{r}} \sum_{k \in N_{i}(z)} h_{k} c_{k}+\sum_{1 \leq j \leq l \leq \bar{r}}\left(\sum_{m \in N_{j}(z), k \in N_{l}(z), m<k} h_{m k}\right) \\
& =\sum_{i=1}^{\bar{r}} \sum_{k \in N_{i}(z)} h_{k} c_{k}+\sum_{1 \leq j=l \leq \bar{r}}\left(\sum_{m \in N_{j}(z), k \in N_{l}(z), m<k} h_{m k}\right)+\sum_{1 \leq j<l \leq \bar{r}}\left(\sum_{m \in N_{j}(z), k \in N_{l}(z), m<k} h_{m k}\right) \\
& =\sum_{i=1}^{\bar{r}}\left(\sum_{k \in N_{i}(z)} h_{k} c_{k}+\sum_{m, k \in N_{i}(z), m<k} h_{m k}\right)+\sum_{1 \leq j<l \leq \bar{r}}\left(h_{m \in N_{j}(z), k \in N_{l}(z), m<k}\right) .
\end{aligned}
$$

Letting $J\left(b_{i}, 1\right):=\left\{s \in \mathcal{J}: s \circ b_{i}=s\right\}$, from Lemma 20 in [5], we know that for all $1 \leq i \leq \bar{r}$, $\left(\sum_{k \in N_{i}(z)} h_{k} c_{k}+\sum_{m, k \in N_{i}(z), m<k} h_{m k}\right) \in J\left(b_{i}, 1\right)$. This means that there exists a Jordan frame $\left\{e_{j}: j \in N_{i}(z)\right\}$ in $J\left(b_{i}, 1\right)$ such that

$$
\left(\sum_{k \in N_{i}(z)} h_{k} c_{k}+\sum_{m, k \in N_{i}(z), m<k} h_{m k}\right)=\sum_{j \in N_{i}(z)} \bar{h}_{j} e_{j} \text { with } b_{i}=\sum_{j \in N_{i}(z)} e_{j} .
$$

Therefore, we obtain

$$
\sum_{i=1}^{\bar{r}}\left(\sum_{k \in N_{i}(z)} h_{k} c_{k}+\sum_{m, k \in N_{i}(z), m<k} h_{m k}\right)=\sum_{i=1}^{\bar{r}} \sum_{j \in N_{i}(z)} \bar{h}_{j} e_{j}, \quad \text { and } z=\sum_{i=1}^{\bar{r}} \sum_{j \in N_{i}(z)} \mu_{i}(z) e_{j},
$$

which implies that

$$
\sum_{i=1}^{\bar{r}}\left(\sum_{k \in N_{i}(z)} h_{k} c_{k}+\sum_{m, k \in N_{i}(z), m<k} h_{m k}\right) \text { and } z \text { operator commute }
$$

because $\left\{e_{j}, j \in N_{i}(z)\right\}(i \in\{1, \cdots, \bar{r}\})$ form a Jordan frame in $\mathcal{A}$ by Proposition 2.6 in [10]. Since $z$ and $h$ do not operator commute, it follows that

$$
\sum_{1 \leq j<l \leq \bar{r}}\left(\sum_{m \in N_{j}(z), k \in N_{l}(z), m<k} h_{m k}\right) \neq 0
$$


Utilizing the linearity of $L(\cdot)$ and the definition of $Q(\cdot)$, we rewrite the above $W$ as

$$
\begin{aligned}
& W=2 \sum_{i \neq j, i, j=1}^{r} \min \left\{\left[\lambda_{i}(z), \lambda_{j}(z)\right]_{g}\right\} L\left(c_{i}\right) L\left(c_{j}\right)+\sum_{i=1}^{r} \min \left\{\left[\lambda_{i}(z), \lambda_{i}(z)\right]_{g}\right\} Q\left(c_{i}\right) \\
& =2 \sum_{i, j=1}^{r} \min \left\{\left[\lambda_{i}(z), \lambda_{j}(z)\right]_{g}\right\} L\left(c_{i}\right) L\left(c_{j}\right)-\sum_{i=1}^{r} \min \left\{\partial g\left(\lambda_{i}(z)\right)\right\} L\left(c_{i}\right) \\
& =2 \sum_{k, l=1}^{\bar{r}} \sum_{i \in N_{k}(z), j \in N_{l}(z)} \min \left\{\left[\lambda_{i}(z), \lambda_{j}(z)\right]_{g}\right\} L\left(c_{i}\right) L\left(c_{j}\right)-\sum_{k=1}^{\bar{r}} \sum_{i \in N_{k}(z)} \min \left\{\partial g\left(\lambda_{i}(z)\right)\right\} L\left(c_{i}\right) \\
& =2 \sum_{k, l=1}^{\bar{r}} \min \left\{\left[\mu_{k}(z), \mu_{l}(z)\right]_{g}\right\} L\left(\sum_{i \in N_{k}(z)} c_{i}\right) L\left(\sum_{j \in N_{l}(z)} c_{j}\right) \\
& -\sum_{k=1}^{\bar{r}} \min \left\{\partial g\left(\mu_{k}(z)\right)\right\} L\left(\sum_{i \in N_{k}(z)} c_{i}\right) \\
& =2 \sum_{k \neq l, k, l=1}^{\bar{r}} \min \left\{\left[\mu_{k}(z), \mu_{l}(z)\right]_{g}\right\} L\left(b_{k}\right) L\left(b_{l}\right)+ \\
& +2 \sum_{k=1}^{\bar{r}} \min \left\{\left[\mu_{k}(z), \mu_{k}(z)\right]_{g}\right\} L\left(b_{k}\right) L\left(b_{k}\right)-\sum_{k=1}^{\bar{r}} \min \left\{\partial g\left(\mu_{k}(z)\right)\right\} L\left(b_{k}\right) \\
& =2 \sum_{k \neq l, k, l=1}^{\bar{r}}\left[\mu_{k}(z), \mu_{l}(z)\right]_{g} L\left(b_{k}\right) L\left(b_{l}\right)+\sum_{k=1}^{\bar{r}} w_{k k} Q\left(b_{k}\right) .
\end{aligned}
$$

Then,

$$
\begin{aligned}
\langle h, W h\rangle & =\left\langle h, 2 \sum_{i \neq j, i, j=1}^{\bar{r}}\left[\mu_{i}(z), \mu_{j}(z)\right]_{g} L\left(b_{j}\right) L\left(b_{i}\right) h\right\rangle+\left\langle h, \sum_{i=1}^{\bar{r}} w_{i i} Q\left(b_{i}\right) h\right\rangle \\
& =\sum_{1 \leq j<l \leq \bar{r}}\left[\mu_{j}(z), \mu_{l}(z)\right]_{g}\left\|\sum_{m \in N_{j}(z), k \in N_{l}(z), m<k} h_{m k}\right\|^{2}+\sum_{i=1}^{\bar{r}} w_{i i}\left\langle h, Q\left(b_{i}\right) h\right\rangle,
\end{aligned}
$$

where the last equality holds by Spectral Decomposition Type I and the fact that

$$
\begin{aligned}
L\left(b_{j}\right) L\left(b_{i}\right) h & =b_{j} \circ\left(b_{i} \circ h\right)=\frac{1}{4} \sum_{m \in N_{j}(z), k \in N_{i}(z), m \neq k} h_{m k} \\
& =\frac{1}{2} \sum_{m \in N_{j}(z), k \in N_{i}(z), m<k} h_{m k} .
\end{aligned}
$$

Using $\left[\mu_{j}(z), \mu_{l}(z)\right]_{g}>0$ for $1 \leq j<l \leq \bar{r}$ and $w_{i i} \geq 0$ for $i \in\{1,2, \cdots, \bar{r}\}$, and applying the inequality $\left\langle h, Q\left(b_{i}\right) h\right\rangle \geq 0$ (see, e.g., [19] ), we derive that

$$
\langle h, W h\rangle=0 \Rightarrow \sum_{1 \leq j<l \leq \bar{r}}\left[\mu_{j}(z), \mu_{l}(z)\right]_{g}\left\|\sum_{m \in N_{j}(z), k \in N_{l}(z), m<k} h_{m k}\right\|^{2}=0 .
$$


Moreover,

$$
\sum_{1 \leq j<l \leq \bar{r}}\left\|\sum_{m \in N_{j}(z), k \in N_{l}(z), m<k} h_{m k}\right\|^{2}=0,
$$

a contradiction to (2). So, in this case, $\langle h, W h\rangle>0$ and hence $\langle h, V h\rangle>0$ for all $V \in \partial G(z)$.

By the mean value theorem, $G(y)-G(x) \in \operatorname{conv}\{\partial G(z)(y-x): z \in[x, y]\}$. Therefore,

$$
\langle y-x, G(y)-G(x)\rangle \in \operatorname{conv}\left\{\langle h, V h\rangle: V \in \bigcup_{z \in[x, y]} \partial G(z)\right\} .
$$

The last inclusion implies in this case that $\langle y-x, G(y)-G(x)\rangle>0, \quad \forall x \neq y$.

Combining the above two cases, we conclude the proof of Part (b).

Part (c): " $\Rightarrow$ " It is similar to the proof of Part (a).

" $\Leftarrow$ " Let $\bar{g}(t):=g(t)-\mu$ for $t \in(a, b)$. Then $\bar{g}(\cdot)$ is monotone on $(a, b)$ by the assumption. Applying the sufficiency result of Part (a), we observe that $\bar{G}(x)$, the corresponding Löwner operator of $\bar{g}(\cdot)$, is monotone on the box set $\mathcal{J}(a, b)$ (it is easy to verify $\bar{G}(x)=G(x)-\mu I)$. The desired conclusion therefore follows immediately.

4. Application: Mangasarian C-function. Recall the Mangasarian class of NCPfunctions $\phi_{M}(a, b)=\theta(|a-b|)-\theta(a)-\theta(b), \forall a, b \in \mathbb{R}$, where $\theta$ is a strictly increasing function from $\mathbb{R}$ into $\mathbb{R}$ with $\theta(0)=0$. Let $\Theta$ denote the corresponding Löwner operator of $\theta$. We define Mangasarian C-function $\Phi_{M}: \mathcal{J} \times \mathcal{J} \rightarrow \mathcal{J}$ by

$$
\Phi_{M}(x, y):=\Theta(|x-y|)-\Theta(x)-\Theta(y) .
$$

By employing Theorem 3, we obtain the following theorem, which shows that $\Phi_{M}$ is a Cfunction for SCCP.

Theorem 4 Let $\mathcal{A}$ be a Euclidean Jordan algebra of rank $r$, and $K$ be the symmetric cone. If $\Phi_{M}$ is given by (3) with $\theta$ being a strictly increasing function from $\mathbb{R}$ into $\mathbb{R}$ with $\theta(0)=0$, then the following statements are equivalent:

(a) $x \in K, \quad y \in K$, and $x \circ y=0$.

(b) $\Phi_{M}(x, y)=0$.

Proof. " $(a) \Rightarrow(b)$ " Since (a) holds, the elements $x, y$ operator commute by Proposition 6 in [5]. Thus, there is a Jordan frame $\left\{e_{1}, \cdots, e_{r}\right\}$ such that $x=\sum_{i=1}^{r} x_{i} e_{i}$ and $y=\sum_{i=1}^{r} y_{i} e_{i}$. So, $x \circ y=\sum_{i=1}^{r} x_{i} y_{i} e_{i}$, and (a) implies that $x_{i} \geq 0, y_{i} \geq 0$ and $x_{i} y_{i}=0$ for all $i \in$ $\{1,2, \cdots, r\}$. Then $\theta\left(\left|x_{i}-y_{i}\right|\right)-\theta\left(x_{i}\right)-\theta\left(y_{i}\right)=0$ for all $i \in\{1,2, \cdots, r\}$. Thus, we have

$$
\begin{aligned}
\Phi_{M}(x, y) & =\Theta(|x-y|)-\Theta(x)-\Theta(y) \\
& =\sum_{i=1}^{r} \theta\left(\left|x_{i}-y_{i}\right|\right) e_{i}-\sum_{i=1}^{r} \theta\left(x_{i}\right) e_{i}-\sum_{i=1}^{r} \theta\left(y_{i}\right) e_{i} \\
& =\sum_{i=1}^{r}\left[\theta\left(\left|x_{i}-y_{i}\right|\right)-\theta\left(x_{i}\right)-\theta\left(y_{i}\right)\right] e_{i}=0 .
\end{aligned}
$$


"(b) $\Rightarrow(a)$ " Let the spectral decompositions of $x-y$ and $|x-y|$ be given by

$$
x-y=\sum_{i=1}^{r} z_{i} e_{i} \text { and }|x-y|=\sum_{i=1}^{r}\left|z_{i}\right| e_{i},
$$

where $\left\{e_{1}, \cdots, e_{r}\right\}$ is a Jordan frame in $\mathcal{A}$. Then, by Theorem 1 we have

$$
x=\sum_{i=1}^{r} x_{i} e_{i}+\sum_{1 \leq j<l \leq r} x_{j l}, y=\sum_{i=1}^{r} y_{i} e_{i}+\sum_{1 \leq j<l \leq r} y_{j l}
$$

where $x_{i}, y_{i} \in \mathbb{R}$, and $x_{j l}, y_{j l} \in J_{j l}$. Furthermore,

$$
\left\langle\sum_{1 \leq j<l \leq r} x_{j l}, u\right\rangle=0, \quad\left\langle\sum_{1 \leq j<l \leq r} y_{j l}, u\right\rangle=0
$$

for any $u \in \operatorname{span}\left\{e_{1}, \cdots, e_{r}\right\}$. Comparing (4) with (5), we obtain that

$$
\sum_{1 \leq j<l \leq r} x_{j l}=\sum_{1 \leq j<l \leq r} y_{j l}
$$

Take $u$ in (6) as $u:=\sum_{i=1}^{r} \theta\left(\left|z_{i}\right|\right) e_{i}-\sum_{i=1}^{r} \theta\left(x_{i}\right) e_{i}-\sum_{i=1}^{r} \theta\left(y_{i}\right) e_{i}$. Then, by the definition of $\Theta$ and $0=\Phi_{M}(x, y)=\Theta(|x-y|)-\Theta(x)-\Theta(y)$, we conclude

$u=\Theta(|x-y|)-\Theta\left(\sum_{i=1}^{r} x_{i} e_{i}\right)-\Theta\left(\sum_{i=1}^{r} y_{i} e_{i}\right)=\Theta(x)+\Theta(y)-\Theta\left(\sum_{i=1}^{r} x_{i} e_{i}\right)-\Theta\left(\sum_{i=1}^{r} y_{i} e_{i}\right)$,

from which we deduce using (6) and (7) that

$$
\begin{aligned}
0 & =\left\langle\sum_{1 \leq j<l \leq r} x_{j l}, u\right\rangle=\left\langle\sum_{1 \leq j<l \leq r} x_{j l}, \Theta(x)-\Theta\left(\sum_{i=1}^{r} x_{i} e_{i}\right)+\Theta(y)-\Theta\left(\sum_{i=1}^{r} y_{i} e_{i}\right)\right\rangle \\
& =\left\langle x-\sum_{i=1}^{r} x_{i} e_{i}, \Theta(x)-\Theta\left(\sum_{i=1}^{r} x_{i} e_{i}\right)\right\rangle+\left\langle y-\sum_{i=1}^{r} y_{i} e_{i}, \Theta(y)-\Theta\left(\sum_{i=1}^{r} y_{i} e_{i}\right)\right\rangle .
\end{aligned}
$$

This along with Theorem 3 and the strict monotonicity of $\theta$ yields $x-\sum_{i=1}^{r} x_{i} e_{i}=0, y-$ $\sum_{i=1}^{r} y_{i} e_{i}=0$. Hence, $|x-y|=\sum_{i=1}^{r}\left|x_{i}-y_{i}\right| e_{i}$ and

$$
0=\Phi_{M}(x, y)=\Theta(|x-y|)-\Theta(x)-\Theta(y)=\sum_{i=1}^{r}\left[\theta\left(\left|x_{i}-y_{i}\right|\right)-\theta\left(x_{i}\right)-\theta\left(y_{i}\right)\right] e_{i} .
$$

Thus, for all $i \in\{1,2, \cdots, r\}$ we have $\theta\left(\left|x_{i}-y_{i}\right|\right)-\theta\left(x_{i}\right)-\theta\left(y_{i}\right)=0$. Note that $\theta$ is strictly monotone on $\mathbb{R}$. The desired conclusion follows immediately from Mangasarian's original result [16].

Acknowledgments We thank three anonymous referees for their very useful comments. The work was supported in part by a Discovery Grant from NSERC, the National Natural Science Foundation of China (10831006) and the National Basic Research Program of China (2010CB732501). 


\section{References}

[1] Baes, M.: Convexity and differentiability properties of spectral functions and spectral mappings on Euclidean Jordan algebras. Linear Algebra Appl. 422, 664-700 (2007)

[2] Faraut, J., Korányi, A.: Analysis on Symmetric Cones. Oxford University Press, New York, 1994

[3] Faybusovich, L.: Euclidean Jordan algebras and interior-point algorithms. Positivity 1, 331-357 (1997)

[4] Facchinei, F., Pang, J.-S.: Finite-Dimensional Variational Inequalities and Complementarity Problems. Volume I and II. Springer-Verlag, New York, 2003

[5] Gowda, M.S., Sznajder, R., Tao, J.: Some $P$-properties for linear transformations on Euclidean Jordan algebras. Linear Algebra Appl. 393, 203-232 (2004)

[6] Huang, Z.H., Ni, T.: Smoothing algorithms for complementarity problems over symmetric cones. Comput. Optim. Appl. 45, 557-579 (2010)

[7] Korányi, A.: Monotone functions on formally real Jordan algebras. Math. Ann. 269, 73-76 (1984)

[8] Kong, L.C., Sun, J., Xiu, N.H.: A regularized smoothing Newton method for symmetric cone complementarity problems. SIAM J. Optim. 19, 1028-1047 (2008)

[9] Kong, L.C., Tunçel, L., Xiu, N.H.: Löwner Operators and Its Applications to Symmetric Cone Complementarity Problems. Research Report CORR 2007-07, University of Waterloo, Ontario, Canada, April 2007

[10] Kong, L.C., Tunçel, L., Xiu, N.H.: Clarke Generalized Jacobian of the Projection onto Symmetric Cones. Set-Valued and Variational Anal.17, 135-151 (2009)

[11] Kong, L.C., Xiu, N.H.: New smooth C-functions for symmetric cone complementarity problems. Optim. Lett. 1, 391-400 (2007)

[12] Lewis, A.S.: Convex analysis on Hermitian matrices. SIAM J. Optim. 6, 164-177 (1996)

[13] Lewis, A.S.: Derivatives of spectral functions. Math. Oper. Res. 21, 576-588 (1996)

[14] Liu, Y., Zhang, L., Wang, Y.: Some properties of a class of merit functions for symmetric cone complementarity problems. Asia-Pac. J. Oper. Res. 23, 473-496 (2006)

[15] Löwner, K.: Über monotone matrixfunctionen. Math. Z. 38, 177-216 (1934)

[16] Mangasarian, O.L.: Equivalence of the complementarity problem to a system of nonlinear equations. SIAM J. Appl. Math. 31, 89-92 (1976)

[17] Rockafellar, R.T., Wets, R.J.-B.: Variational Analysis. Springer, New York, 2004

[18] Sun, D., Sun, J.: Löwner's operator and spectral functions on Euclidean Jordan algebras. Math. Oper. Res. 33, 421-445 (2008) 
[19] Sturm, J.F. 2000. Similarity and other spectral relations for symmetric cones. Linear Algebra Appl. 312 135-154.

[20] Tseng, P.: Merit function for semi-definite complementarity problems. Math. Program. 83, 159-185 (1998)

[21] Yoshise, A.: Interior point trajectories and a homogeneous model for nonlinear complementarity problems over symmetric cones. SIAM J. Optim. 17, 1129-1153 (2006) 